te untersucht. Diese Untersuchungen und die Ergebnisse, zu denen die Autorin kommt, sind unterstützt und belegt durch insgesamt 120 Tabellen und 85 Abbildungen, die für das Verständnis außerordentlich hilfreich, geradezu unerlässlich sind.

Die Ergebnisse dieser Untersuchungen sind, gerade weil sie in jedem Einzelfall durch Statistiken und zahlreiche Fallbeispiele mit Musterrechnungen bewiesen sind, erschütternd. Hier nur kurz beispielhaft Einiges:

So liegen die Unterhaltstabellenbeträge regelmäßig unter dem Existenzminimum entsprechend dem Existenzminimumbericht der Bundesregierung. Z.B. findet sich in der DT vom 1. Juli 2001 das durchschnittliche kindliche Existenzminimum entsprechend dem 3. Existenzminimumbericht erst in Einkommensgruppe 5 (!).

2001 ist mit Auswertung der Einkommens- und Verbrauchsstichprobe des Statistischen Bundesamts festzustellen, dass 55 Prozent der erfassten und 66 Prozent der hochgerechneten Haushalte von Alleinerziehenden mit einem oder mehreren Kindern Unterhalt in einer Höhe erhalten, die nicht einmal den Mindestunterhalt für ein Kind deckt.

Bereits die Wahl der Normfamilie (zwei Erwachsene und zwei Kinder bzw. seit 2010 zwei Erwachsene und ein Kind), obwohl die Normfamilie von Unterhaltsberechtigten aus einem Erwachsenen mit einem oder mehreren Kindern besteht, begünstigt im Ergebnis den Unterhaltsberechtigten, wie durch Vergleichsberechnungen nachgewiesen wird.
Deutlich wird auch immer wieder die Privilegierung der Unterhaltspflichtigen in höheren Einkommensgruppen, de facto eine „Schonung der regelsetzenden Schicht“. Ein Erfolgsgeheimnis ist dabei das „Spielen mit den Einkommensgruppen“, das in der Regel Unterhaltserhöhungen verhindert.

Selbstbehaltsbeträge täuschen eine Unterhaltslast vor, die es so nicht gibt, weil den Unterhaltspflichtigen in der Regel weit mehr als der Selbstbehalt bleibt.

Das Zusammenspiel von Erhöhung der Selbstbehaltsbeträge und geringfügig erhöhter Kindesunterhaltsbeträge verhindert dabei häufig eine tatsächliche Erhöhung des Kindesunterhalts, da Abänderungsklagen zumindest riskant erscheinen.

Ein „zivilrechtliches Existenzminimum“ in Form des Bedarfskontrollbetrages existiert nur für den Unterhaltspflichtigen, nicht für die Kinder.

Mit einer Fülle von Einzelbeispielen macht die Autorin sichtbar, dass die DT keineswegs ein neutrales, objektives Instrument zur Herstellung von Verteilungsgerechtigkeit ist, sondern ein zutiefst tendenziöses, wobei die Tendenz eben häufig nicht offensichtlich ist, sondern es tatsächlich der Statistiken und Berechnungen der Autorin bedarf, um dies zweifelsfrei zu belegen.

Ein dringend notwendiges, außerordentlich spannendes und lehrreiches Buch und hoffentlich ein Beitrag auf dem Weg von der scheinbaren Tabellengerechtigkeit hin zur größeren Einzelfallgerechtigkeit.

\title{
Rezension:
}

\section{Männlich inszenierte Elite}

\section{Über die Rolle des Journalismus als Konstrukteur geschlechtsgebundener Berichterstattung}

\section{Katrin Lange}

Projektmitarbeiterin, Deutscher Juristinnenbund e.V., Berlin

Lünenborg, Margreth / Röser, Jutta (Hrsg.):

Ungleich Mächtig. Das Gendering von Führungspersonen aus Politik, Wirtschaft und Wissenschaft in der Medienkommunikation, 2012.

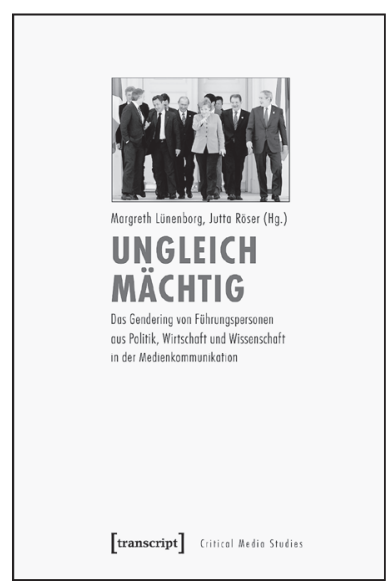

In ihrem Buch „Ungleich mächtig " nehmen die Autorinnen ${ }^{1}$ die mediale Konstruktion von Macht und Geschlecht in den Bereichen Politik, Wirtschaft und Wissenschaft ins Visier. Anhand eines „integrativen Forschungsdesigns“ (S. 7 ff.), das verschiedene Formen der Medien- und Kontextanalysen integriert, wird der Frage nachgegangen, wie Frauen und Männer in Spitzenpositionen visuell und sprachlich sichtbar gemacht und ob beziehungsweise wie diese Darstellungen geschlechtsgebunden formuliert werden. Die Rolle der Medien sei von zentraler Bedeutung, da „Vorstellungen von Spitzenkräften aus Politik, Wirtschaft und Wissenschaft wesentlich auf Basis der Medienberichterstattung “ (S. 22) beruhten und folglich Medien mitgestalten, „wie Frauen und Männer in diesen gesellschaftlichen Teilbereichen sein sollen und was als nicht wünschenswert gilt“ (S. 67).

Nach einem kurzen Einführungskapitel werden im ersten Beitrag die Ergebnisse der quantitativen Inhaltsanalyse präsentiert. Im Fokus steht hier eine statistische Auszählung, wie häufig über Frauen und Männer in Spitzenpositionen in den Medien berichtet wird. Das Ergebnis - „nicht einmal jede fünfte Person aus Politik, Wirtschaft und Wissenschaft, über die berichtet wird, ist weiblich“ (S. 44) - ist wenig

1 Elke Grittmann, Margreth Lünenborg, Tanja Maier, Kathrin Frederike Müller und Jutta Röser. Bereits 2011 wurde der Artikel „Zur medialen Sichtbarkeit von wirtschaftlichen Spitzenkräften“ von Margreth Lünenborg und Tanja Maier in der Studie des djb „Aktionärinnen fordern Gleichberechtigung - Erhöhung des Frauenanteils in Führungspositionen im europäischen Kontext" veröffentlicht. 
überraschend und bestätigt die bereits bestehende Vermutung der weiblichen Unterrepräsentanz in den Medien. Einziger Lichtpunkt ist der sogenannte „Kanzlerin-Effekt“, der durch die häufige Präsenz Angela Merkels in den Medien die mediale Sichtbarmachung anderer Frauen positiv beeinflusse.

Der zweite Beitrag untersucht, in Form einer qualitativen Inhaltsanalyse, nicht „wie oft“, sondern „wie“ Personen medial präsentiert werden. Dem liegt ein grundlegendes Verständnis von Journalismus als Konstrukteur eigener Realitäten zugrunde: In den Medien ist Wirklichkeit nicht Abbild, sondern Bezugspunkt mit der Folge, dass Wirklichkeit interpretiert, gestaltet oder sogar erst konstruiert werden kann. Positiv festzuhalten ist, dass Frauen wie Männer in allen drei gesellschaftlichen Teilbereichen als erfolgreich und kompetent beschrieben werden und sogar Anhaltspunkte dafür gefunden werden, „dass Geschlechterverhältnisse in Mediendiskursen im Wandel sind“ (S. 114). Dennoch fällt die mediale Beschreibung von Weiblichkeit stets hinter die von Männlichkeit, welche nach wie vor als „Norm“ (S. 115) fungiert, zurück. Zu oft werden „der Einfachheit wegen “, so die Erklärung, altbekannte Muster der Geschlechtszuschreibung verwendet. Überhaupt funktioniert die mediale Darstellung weiblicher Personen in den Führungsriegen von Politik, Wirtschaft und Wissenschaft ausschließlich über ihre Verbindung zum und Herausstellung von Geschlecht. Äußerlichkeiten (Körper, Kleidung, Frisur etc.) würde bei der Beschreibung von Frauen ein höherer Rang eingeräumt als bei der von Männern; und schlimmstenfalls ist weiblicher Erfolg in der Berichterstattung nur darüber erklärbar, dass „der Frau doch noch ein Mann zur Seite steht“ (S. 106). Der Wandel, so schlussfolgern die Autorinnen am Ende, beziehe sich letztlich doch nur auf die Beschreibung von Weiblichkeit im Journalismus, die keine Züge offener Diskriminierung und Abwertung beinhalte - während die mediale Beschreibung von Männlichkeit(en) eine gewisse Statik aufweise (S. 115) und in ihrem Verharren auch die Grenzen des Wandels der Geschlechterverhältnisse festlege.

Im dritten Beitrag werden die bisherigen Ergebnisse durch Analyse der visuellen Berichterstattung in Form einer quantitativen und qualitativen Bildanalyse untermauert: Nicht nur die zunehmende Bedeutung visueller Berichterstattung, sondern auch ihre Besonderheit, dem Eindruck nach „Tatsächliches“ anschaulich festzuhalten, rücke visuelle Medien in den Fokus; bezüglich des Verhältnisses von Geschlecht und Macht, so die Prämisse, „eignet sich damit das Medium wie kein anderes dazu, Geschlechterdifferenzen zu naturalisieren“ (S. 130). In ihrer Analyse kommen die Autorinnen folglich auch zu dem Ergebnis, die Befunde der Bildanalyse deckten sich weitgehend mit denen der Textanalyse, wobei die visuelle Berichterstattung in den drei gesellschaftlichen Teilbereichen eine jeweils eigene Darstellungsweise von Spitzenpersonal in Handlungskontexten entwickelt habe - damit führen die Autorinnen allerdings eine separate Betrachtung durch und werden dem Anspruch einer vergleichenden Perspektive von Politik, Wirtschaft und Wissenschaft als eingangs formuliertem
„Alleinstellungsmerkmal“ dieser wissenschaftlichen Arbeit nicht gerecht. Potential sehen die Autorinnen darin, dass tradierte Bilder von Weiblichkeit wie das Bild der „First Lady“, der „Frau an seiner Seite“, der „fürsorglichen Mutter“ oder der „Tochter des Unternehmers“ zunehmend in Konflikt gerieten mit neuen Formen medialer Sichtbarkeit von Frauen, die einhergehe mit „einer größeren Handlungsfähigkeit und Machtzuschreibung von Frauen in Spitzenpositionen“ (S. 164).

Die Ergebnisse der vorangegangenen Analysen führen die Autorinnen im vierten Beitrag zur folgerichtigen Annahme, weibliche Unterrepräsentanz in den Medien sei nicht allein auf den geringeren Frauenanteil in Führungspositionen in der Gesellschaft zurückzuführen; vielmehr geraten mediale Mechanismen - die Art und Weise, wie Berichterstattung funktioniert, welchen Mustern, Regeln und Routinen sie dabei folgt in den Fokus. Mittels Experteninterviews wurden acht Journalistinnen und Journalisten zu ihrer Arbeit befragt, wobei die anfangs mehrheitlich formulierte Sichtweise der Befragten bezüglich der Aufgabe des Journalismus als Medium, welches Wirklichkeit lediglich abbildet, auffällt: Dies widerspricht der im zweiten Beitrag formulierten Annahme, Journalismus gestalte Wirklichkeit „mit“, wenn er sie nicht gar mitkonstruiere. Die Befragten sehen sich zudem in ihrer journalistischen Tätigkeit eher als „Spielfiguren“ in einem Spannungsfeld zwischen Leserinteressen und gesellschaftlichen Machtzentren, insbesondere dem der Politik. Die Autorinnen weisen anhand dieser getätigten Aussagen nach, dass eher unreflektiert Personen ausgewählt und über diese dann in einem Beitrag berichtet werde; oftmals sei der in den Medien gern herangezogene „Experte“ sogar „selbst gemacht“: durch wiederholtes mediales Verweisen auf eben jene „Expertise“. Frauen ziehe man in Betracht, wenn deren Fehlen allzu offensichtlich werde, beispielsweise bei der Zusammensetzung einer Talkrunde, was „die vermeintliche „natürliche“ Autorität der männlichen Expertise [jedoch] nicht in Zweifel“ (S. 186) ziehe. Auch habe in der Vergangenheit der steigende Anteil an Frauen in den Redaktionen nicht dazu geführt, dass über Frauen häufiger berichtet beziehungsweise eine stereotype Beschreibung von Weiblichkeit und Männlichkeit aufgegeben werde. Die Beschäftigung mit „Frauenthemen“ werde mitunter als „Abwertung der eigenen journalistischen Arbeit begriffen" (S. 107). Allein die Identifikation eines „Frauenthemas“ sei eine mit Geschlecht verknüpfte Zuschreibung, die selten reflektiert werde - auch Dreh- und Angelpunkt, um Journalistinnen und Journalisten für eine geschlechtersensiblere Berichterstattung zu gewinnen: Nur wenn erkannt werde, dass der eigene Beitrag bestehende gesellschaftliche Verhältnisse fortschreibt, eröffne sich ein Handlungsspielraum, um „Normen zu verschieben und Wirklichkeitskonstruktionen zu verändern“ (S. 199). Letztlich gelte dies nicht nur für diejenigen, die journalistische Texte produzieren, sondern auch für diejenigen, die sie lesen.

Im letzten Beitrag wird so die Rolle der Mediennutzerin beziehungsweise des Mediennutzers in den Blick genommen und untersucht, ob und wie geschlechtsgebundene Bericht- 
erstattung wahrgenommen wird. Als Ergebnis kann die so genannte „Vorzugslesart“ (S. 226) von Frauen festgehalten werden, „weil sich diese [...] gegen Widerstände von unten nach oben gekämpft haben und deshalb primär nicht für die Ausübung von Macht stehen“ (ebd.). Macht sei Gebiet des Mannes, womit die in den wissenschaftlichen Analysen ausgemachte „Norm“ bestätigt werde. Das Vorhandensein weiblicher Führungskräfte in diesem Gebiet finde zwar Respekt und Zustimmung, ihr Erfolg werde aber vorrangig über die Aneignung männlich konnotierter Eigenschaften erklärt. Die Frau bleibe somit „Exotin“. Darüber hinaus werde der Erfolg individualisiert und folglich strukturelle Ursachen sozialer Ungleichheit ausgeblendet, obwohl letztere gleichzeitig innerhalb der geführten Gruppendiskussionen erkannt wurde.

Das Buch „Ungleich mächtig“ gibt einen umfassenden Einblick in die Funktionsweise der deutschen Medienlandschaft in Hinblick auf die Konstruktion von Geschlecht. Wesentliche Erkenntnisse der Studien zirkulierten zwar vorher bereits als Vermutungen in der öffentlichen Diskussion über die weibliche Unterrepräsentanz, werden nun aber wissenschaftlich fundiert. Gerade die Berücksichtigung der verschiedenen Blickwinkel in der Analyse führt somit zu einer umfangreichen Bestandsaufnahme.
$\mathrm{Zu}$ kritisieren bleibt, dass der eingangs formulierte Anspruch, „erstmalig [...] mit den Bereichen Politik, Wirtschaft und Wissenschaft drei gesellschaftliche Felder und deren unterschiedliche Repräsentationen vergleichend in den Blick [zu nehmen]“ (S. 7), nicht erfüllt werden kann. Stets fallen vor allem Wissenschaft, aber auch Wirtschaft, mangels zu erhebender Daten, im Vergleich hinter die Analyse der Politik zurück. Unberücksichtigt bleibt auch der Einbezug neuer Medienformate (Blogs, Mags, Social Media-Anwendungen, Kommentarfunktionen etc.) durch das Internet, die eher ein Aufbrechen medial konstruierter Geschlechterverhältnisse vermuten lassen als die analysierten klassischen Medien.

Dies schmälert letztlich aber nicht die durch die Lektüre des Buches gewonnenen Einsichten. Gerade für die Arbeit des djb im Rahmen des Projekts „Aktionärinnen fordern Gleichberechtigung“ ist es von großem Interesse, sein Anliegen - die Erhöhung des Frauenanteils in Führungspositionen in der Wirtschaft - in einen gesamtgesellschaftlichen Kontext zu bringen. Mit klaren Statements kann so der permanenten Verortung in der „Frauensparte“ etwas entgegengesetzt werden, wovon sich im Idealfall auch die anderen 50 Prozent der Gesellschaft angesprochen fühlen.

\section{Das Deutsche Anwaltsinstitut e. V. sucht Referentinnen}

Ungleich zu vielen anderen Berufen sind Frauen in der Anwaltschaft heute keineswegs unterrepräsentiert. Der Frauenanteil ist hier während der letzten 40 Jahre von 4,5 Prozent auf 32 Prozent gestiegen. Auch in Zukunft geht der Aufwärtstrend erst einmal im gleichen Tempo weiter. Bereits im Wintersemester 2004/2005 waren mehr als 50 Prozent der JuraStudierenden Frauen. Inzwischen bewegt sich deren Anteil unaufhaltsam auf die 60-Prozent-Marke zu.

Diese Tendenz findet sich auch in den Teilnehmerzahlen des Deutschen Anwaltsinstituts e. V. (DAI) wieder, der Ausund Fortbildungseinrichtung von Bundesrechtsanwaltskammer, Bundesnotarkammer und 43 Rechtsanwaltskammern und Notarkammern und eines der bundesweit führenden Fortbildungsinstitute für Rechtsanwälte, Fachanwälte, Notare und deren Nachwuchs sowie Mitarbeiter. Bei den unter 35-Jährigen sind es über 50 Prozent Frauen, die an den Seminaren, Fachlehrgängen und Arbeitstagungen des Deutschen Anwaltsinstituts teilnehmen. Diese Zahl schließt sowohl die eigenen Aus- und Fortbildungsstätten in Bochum, Berlin und Heusenstamm bei Frankfurt am Main ein als auch Teilnehmerstatistiken aus mehr als 50 Orten in Deutschland, in denen das Deutsche Anwaltsinstitut seine insgesamt 700 Veranstaltungen mit rund 30.000 Teilnehmerinnen und Teilnehmern jährlich durchführt.

Diesem erfreulichen Aspekt bei der Teilnehmerentwicklung steht jedoch leider eine gähnende Leere in den Rängen von
Referentinnen gegenüber. Denn wie eine Überprüfung des DAI ergeben hat, mangelt es in lehrenden Institutionen einschließlich der juristischen Fakultäten der Hochschulen nach wie vor an Referentinnen und Dozentinnen. Der Anteil der Referentinnen in Aus- und Fortbildungsinstitutionen liegt lediglich bei rund 11 Prozent, in den juristischen Fakultäten der Universitäten bei knapp 16 Prozent, Tendenz sinkend.

Das DAI möchte daher das zweifellos vorhandene, aber bisher weitgehend ungenutzte Potential von Referentinnen für seine Veranstaltungen mit einer bundesweiten Initiative aktivieren und dazu motivieren, erworbene juristische Kenntnisse und Erfahrungen in einem seriösen und effektiven Rahmen an die nächste Generation von Rechtsanwälten und Notaren weiterzugeben. Gesucht werden Anwältinnen, Notarinnen, Richterinnen und Professorinnen, die als Referentinnen im DAIFortbildungsprogramm neue Impulse setzen wollen.

\section{Bitte kontaktieren Sie:}

Dr. Katja Mihm

Geschäftsführerin

Rechtsanwältin, Fachanwältin für Arbeitsrecht, Mediatorin

Deutsches Anwaltsinstitut e. V.

Universitätsstr. 140

44799 Bochum

Tel.: 02349706411

E-Mail:mihm@anwaltsinstitut.de 